\title{
EDITORIAL
}

\section{SUCCESSORS OF ARF WATER-IMMERSION LITHOGRAPHY: EUV LITHOGRAPHY, MULTI-E-BEAM MASKLESS LITHOGRAPHY, OR NANOIMPRINT?}

ArF water-immersion lithography supports a numerical aperture (NA) of 1.35 or slightly higher but cannot reach the theoretical limit of I.44 NA. It is increasingly difficult to resolve half pitches below $k_{1}=0.28$ unless one resorts to splitting the pitch by multiple exposures or multiple patterning. In principle, smaller pitches could always be resolved by more multiplicity, if cost were not an issue. Unfortunately, cost is indeed an issue. Cost dictates whether the product is marketable compared with the previous generation. Cost-effective solutions have to be found either by limiting the number of imaging layers that require multiple patterning or by a less expensive successor to ArF water-immersion lithography.

EUV seems to be the heir apparent, carrying the momentum of photon beams and a reversal of $k_{\text {I }}$ reduction. It has ample funding and many faithful followers. There are only a few countable obstacles to overcome, namely, source power and efficiency, sensitive highresolution low-line-width-roughness (LWR) resists, cost and size of tools, wall power requirement, mask blank, mask inspection and repair, in situ mask pellicle, mirror lifetime, stray light, and thermal management. A high level of funding is good for accelerating the technology if it does not turn into bleeding. It is wise not to drag on the funding to prevent the unbearable recuperation of the development cost through the tool cost.

Multi-e-beam maskless lithography is gaining some momentum but is still a poor stepchild. E-beam lithography has the unfortunate reputation of low throughput despite its ability to be steered with extremely high speed compared to photon beams. The problem is in the individualism of e-beams compared with the mass parallelism of photon beams. Advances in inexpensive e-beam minicolumns, the MEMS technique to produce massively parallel beams inexpensively, or the CMOS technique to create millions of rapid-switching pixels inexpensively, are now introducing parallelism to e-beam systems. The high-speed steering property can now be fully taken advantage of. For the first time, the cost burden of expensive optical components is switched to data-handling electronics. The electronic industry finally has the opportunity to control the cost of its own equipment. Cost reduction dictated by Moores law has a positive feedback to enable more cost reduction. Multi-e-beam maskless lithography has its own list of obstacles, namely, cost, size, and thermal management of data handling, control of massive parallelism, data rate, data integrity, electromagnetic interference shielding, beam consistency, and sensitive high-resolution low-LWR resists.

How about nanoimprint, the lensless replication technique that seems to have unlimited resolution capability and costs pennies for the molding tool? It has taken contact printing to a higher level. Instead of letting the mask contact the photoresist but still occupy its own space, the imaging medium is mingled with the molding template that used to be called the mask. Veterans of contact printing know its vulnerability to defects. Mingling the medium with the mold does not seem to offer more promise for less defects. The mold may have a much shorter lifespan than the optical mask, but child molds can be replicated effortlessly using the same molding technique. Why stop at children? Grandchildren can also be adopted. The childless optical mask needs only one space for storage. Children and grandchildren have to be stored and managed. The template polarity is changed from mother to children, ditto for grandchildren. The preferred stud polarity is reversed to the less preferred pit polarity. Molding is a paced process. Waiting for bubbles to dissolve before molding takes time. Waiting for the fluid to solidify takes time. Pulling out the mold with minimal damage takes time. Producing a uniform residual film under the bottom of the template does not take time but rather stringent specs for the flatness and roughness of the mold and the wafer as well as the forbiddance of nanoparticles under the template.

Which technology will be the successor of ArF waterimmersion lithography? The path of succession is not yet clear. It opens the door for more studies, developments, sharing, and archiving through journal publications. Submit your findings and assertions. $\mathrm{JM}^{3}$ will give you a fair chance to state your case.

Happy reading!

Burn J. Lin

Editor-in-Chief

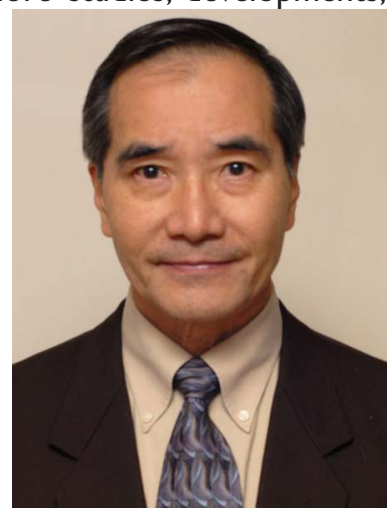

